\title{
Visualization of Acoustic Waves and Cavitation in Ultrasonic Water Flow
}

\author{
Hidehisa Usui ${ }^{1, a}$, Tomoatsu Ishibashi ${ }^{2, b}$, Hisanori Matsuo ${ }^{2, c}$ \\ Katsuhide Watanabe ${ }^{2, \mathrm{~d}}$, and Keita Ando $\mathrm{A}^{1, \mathrm{e}^{*}}$ \\ ${ }^{1}$ Keio University, 3-14-1 Hiyoshi, Kohoku-ku, Yokohama, Kanagawa 223-8522, Japan \\ 2Ebara Corporation, 4-2-1 Honfujisawa, Fujisawa, Kanagawa 251-8502, Japan \\ ahidehisa007@keio.jp, bishibashi.tomoatsu@ebara.com, 'cmatsuo.hisanori@ebara.com \\ dwatanabe.katsuhide@ebara.com, ${ }^{\mathrm{e} k a n d o @ m e c h . k e i o . a c . j p ~}$ \\ ${ }^{*}$ Corresponding author
}

Keywords: Ultrasonic water flow cleaning, Acoustic cavitation, Bubble motion

\begin{abstract}
Visualization experiments were performed to examine whether acoustic bubbles play a role in ultrasonic water flow cleaning, as in convention cleaning with ultrasonic baths. Schlieren visualization confirmed the standing-wave-like acoustic field in ultrasonic water flow that collides with a glass surface. Backlight visualization showed that cavitation bubbles appear in the water flow spreading over the glass surface. These bubbles are found to oscillate in volume and move inside film flow and thus expected to play a role as active cleaning agents.
\end{abstract}

\section{Introduction}

In semiconductor industry, the post-CMP cleaning is an important process, for its performance is one of the key factors in determining the performance of semiconductors. Cleaning with water jets in which ultrasonic waves with frequency of order $1 \mathrm{MHz}$ (the so-called megasonic waves) propagate in their flow direction is recently considered as a potential candidate used in the cleaning process; we call it "ultrasonic water flow cleaning" in this paper. While reattachment of removed particles can be a technical issue in conventional cleaning with ultrasonic cleaning baths, such an issue can be avoid in the case of ultrasonic water flow cleaning, for particle-free water is continuously supplied to cleaning targets. In the experiment of Hauptmann et al. [1], the acoustic field in $1 \mathrm{MHz}$ ultrasonic water flow was visualized by a schlieren technique and the ultrasound-induced cavitation bubbles were detected base on sonoluminescence imaging. We speculate that cavitation bubble dynamics will play a major role in removing surface-attached contaminants as in the case of ultrasonic cleaning baths [2,3]. For a better understanding of the role of acoustic bubbles in ultrasonic water flow cleaning, visualization of ultrasound-induced phenomena including cavitation is essential.

In this study, we aim to obtain a direct evidence of the appearance of cavitation bubbles in ultrasonic water flow and the subsequent spreading flow over a flat glass surface. Using schlieren and backlight methods with a high-speed camera, we observe the acoustic field and cavitation bubbles in the ultrasonic water flow, respectively.

\section{Experimental Method}

The experimental and optical setups for visualization of acoustic fields and cavitation bubbles in ultrasonic water flow are presented in figures 1 and 2, respectively. As depicted in figure 1 (a), the experimental setup consists of an ultrasonic water flow cleaner (QUAVA Spot 27220, KAIJO), a water container, a pump, volume flow meter, and a glass plate (as a cleaning target). Air-saturated tap water approximately at $20^{\circ} \mathrm{C}$ was pumped to the ultrasonic water flow cleaner whose nozzle head is equipped with a piezoelectric transducer driven at input power $50 \mathrm{~W}$ and either at frequency 0.43 or $0.95 \mathrm{MHz}$; the intent of selecting two different ultrasound frequency is to examine frequency dependence on acoustic cavitation. The volume flow is set at $Q=3.3 \times 10^{-5} \mathrm{~m}^{3} / \mathrm{s}$. the average velocities, which is defined as the volume flow divided by the nozzle cross-sectional area $\left(50 \mathrm{~mm}^{2}\right.$ for $0.43 \mathrm{MHz}$ and $38 \mathrm{~mm}^{2}$ for $0.95 \mathrm{MHz}$ ), are computed at $0.66 \mathrm{~m} / \mathrm{s}$ for $0.43 \mathrm{MHz}$ and $0.87 \mathrm{~m} / \mathrm{s}$ for 
$0.95 \mathrm{MHz}$. The corresponding Reynolds numbers are 5600 for $0.43 \mathrm{MHz}$ and 6000 for $0.95 \mathrm{MHz}$, meaning that the flow is turbulent. The ultrasonic water flow is ejected downward from the nozzle to the glass surface. Since the water jet interface is curved, acoustic phenomena including cavitation cannot be observed due to light refraction. In order to visualize those inside the water jet, a glass tube of square cross section (figure 1 (b)) is inserted below the nozzle exit. The cross-sectional area of the tube flow is set at that of the nozzle.

A schlieren method (figure 2 (a)) with the square glass tube is applied to visualize the ultrasonic wave propagation in the water jet; a high-speed camera (FASTCAM SA-X2, Photoron) at $10000 \mathrm{fps}$ is used to record evolution of the acoustic phenomenon, together with a pulse laser stroboscope (CAVILUX smart, Cavitar) at wavelength $640 \mathrm{~nm}$ and duration $10 \mathrm{~ns}$. A backlight method (figure 2 (b)) with the high-speed camera at frame rate $20000 \mathrm{fps}$, a magnification lens (one pixel equal to $10 \mu \mathrm{m}$ ) and LED illumination (SLG-150V, REVOX) is applied to observe acoustic cavitation bubbles in the water jet. From an application point of view, the spreading water as a result of the jet impingement onto the glass surface is visualized as depicted in figure 2 (c), which allows for seeing whether cavitation bubbles appear at the surface. In the radially spreading water film flow, a hydraulic jump occurs. Our observation location is the thin water film flow (of thickness $\approx 1 \mathrm{~mm}$ ) before the hydraulic jump. The backlight method with the high-speed camera at frame rate $30000 \mathrm{fps}$, the magnification lens (one pixel equal to $1 \mu \mathrm{m}$ ) and the pulse laser stroboscope is also used to observe cavitation bubbles in the water film flow.

(a)

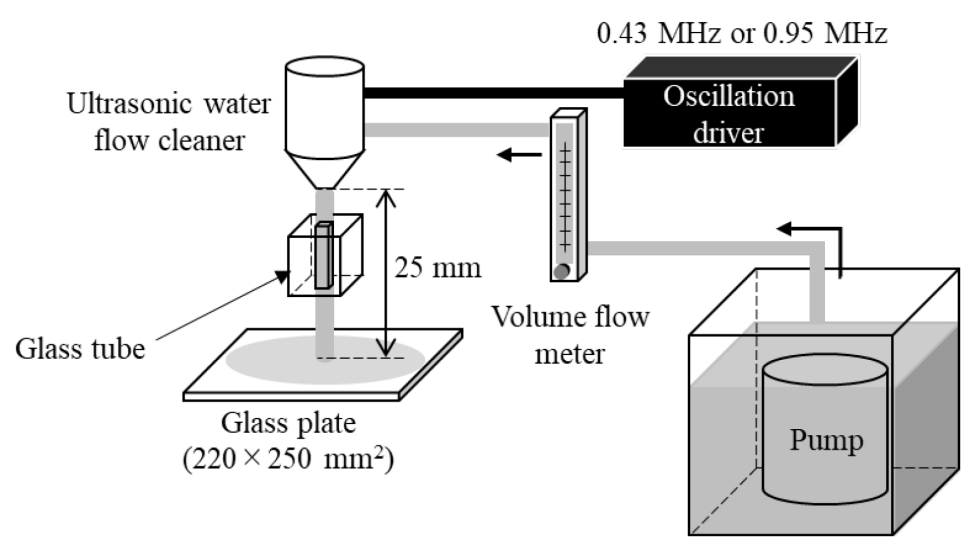

(b)

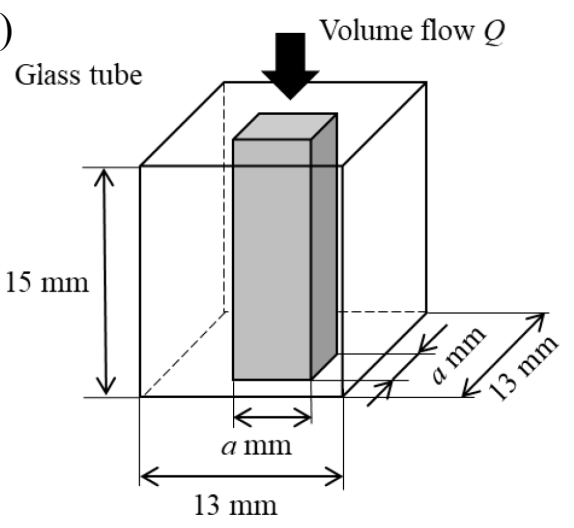

Figure 1: Schematic of the experimental setup: (a) System of an ultrasonic water flow and a glass surface (as a cleaning target), (b) Square glass tube used for visualization inside the water jet. The lengths of side $a$ are $7.19 \mathrm{~mm}$ at $0.43 \mathrm{MHz}$ and $6.15 \mathrm{~mm}$ at $0.95 \mathrm{MHz}$. 


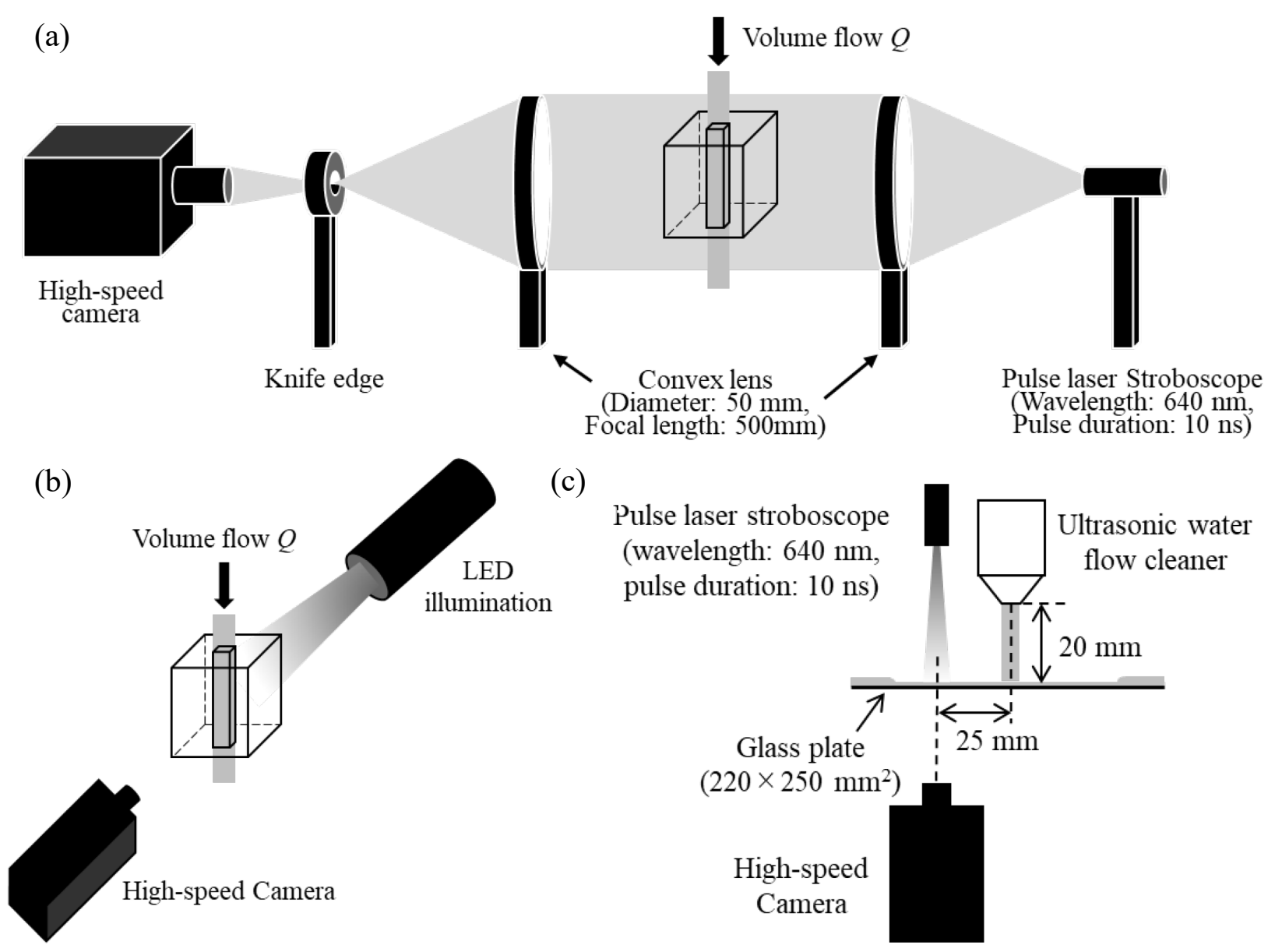

Figure 2: Schematic of the optical setup: (a) Schlieren method for visualization of the acoustic field in the water jet, (b) Backlight method for visualization of cavitation bubbles in the water jet, (c) Backlight method for visualization of cavitation bubbles in the spreading water film flow over the glass surface. The hydraulic jump appears approximately at $50 \mathrm{~mm}$ from the stagnation point of the jet collision against the glass surface.

\section{Results and Discussion}

From a snapshot of schlieren visualization (figure 3), we can see light and dark stripes that are repeated at the interval of $4.0 \pm 0.5 \mathrm{~mm}$ for $0.43 \mathrm{MHz}$ and $1.8 \pm 0.3 \mathrm{~mm}$ for $0.95 \mathrm{MHz}$. Since the wavelengths of 0.43 and $0.95 \mathrm{MHz}$ ultrasound are 3.47 and $1.56 \mathrm{~mm}$, respectively, these stripes will represent sinusoidal waves associated with the ultrasound propagation. We can judge whether these acoustic fields are traveling or standing wave from motion of cavitation bubbles visualized by the backlight technique; see figure 4 . The radii of the oscillating bubbles are found to be roughly $15 \mu \mathrm{m}$ for $0.43 \mathrm{MHz}$ and $10 \mu \mathrm{m}$ for $0.95 \mathrm{MHz}$. These bubbles sizes are close to the resonant bubble radii of Minnaert for air bubbles [3, 4, 5, 6]; cavitation bubbles become smaller with increasing the ultrasound frequency, as expected. Interestingly, some bubbles are advected with the water flow, but others are trapped at the observation spot. The bubble trapping (against fluid-dynamic drag) will be supported by the primary Bjerknes force [7] that appears in a standing acoustic field. This suggests that the standing-wave-like acoustic field is built as a result of superposition of the incident wave and the reflected wave (from the glass surface).

Next, we examine the visualization of the liquid film flow over the glass surface. In figure 5, we can also observe cavitation bubbles in the water film flow over the glass surface for the case of the $0.43 \mathrm{MHz}$ ultrasound. It follows from figure 5 (a) that the film thickness is non-uniform as a result of surface wave propagation (imaged as the fluctuation in the image darkness). This means that the acoustic field inside the liquid film is unsteady due to acoustic wave interaction with the surface waves. After the surface wave (represented by the darker domain in these images) passed by, 
cavitation bubbles can be seen more clearly. It is interesting to see that some bubbles are trapped at specific spots and others are advected with random trajectories. This is perhaps because of the Bjerknes force arising from the unsteady acoustic field inside the liquid film. Such bubbles oscillating in volume and moving around above the glass surface are expected to play a major role in ultrasonic water flow cleaning. We can see the similar result for the case of the $0.95 \mathrm{MHz}$ ultrasound; see figure 6. In this higher frequency case, cavitation bubbles become smaller in comparison to those in the lower frequency case, which is consistent with the observation in figure 4 .
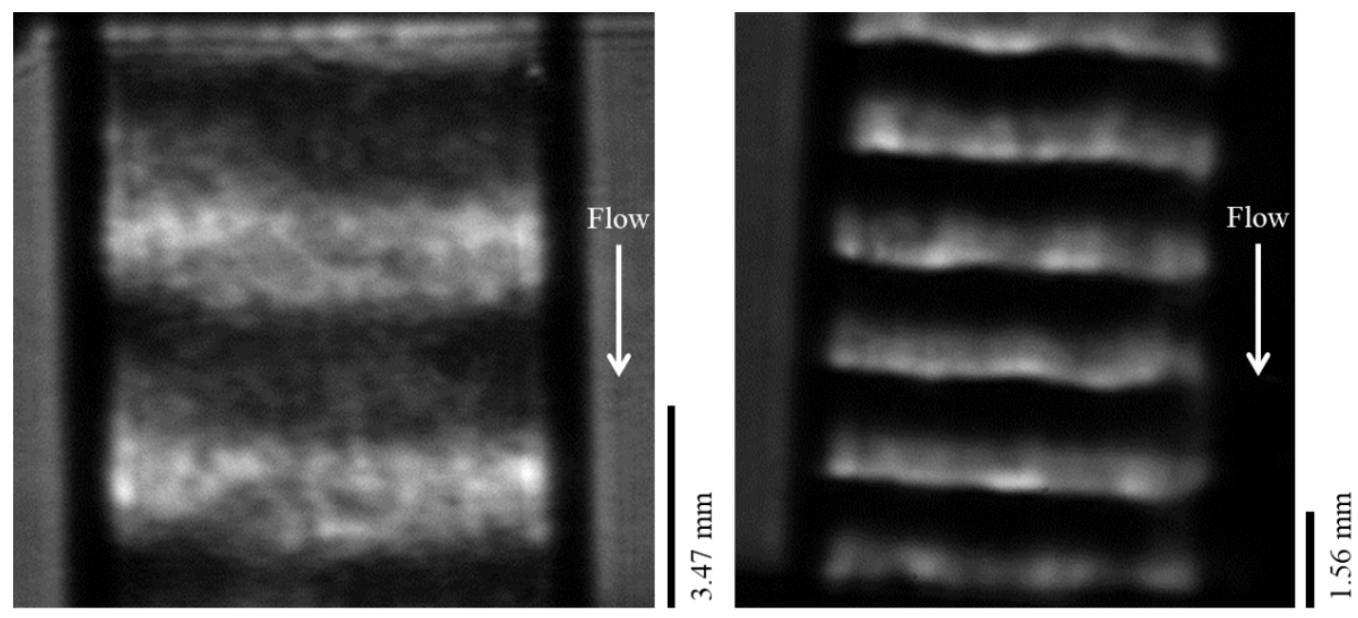

Figure 3: An example of schleiren visualization in the $0.43 \mathrm{MHz}$ (left) and $0.95 \mathrm{MHz}$ (right) ultrasonic water flow through the square glass tube. The scale bars (left, right) equal to the wavelengh of 0.43 and $0.95 \mathrm{MHz}$ ultrasound, respectively.

(a)
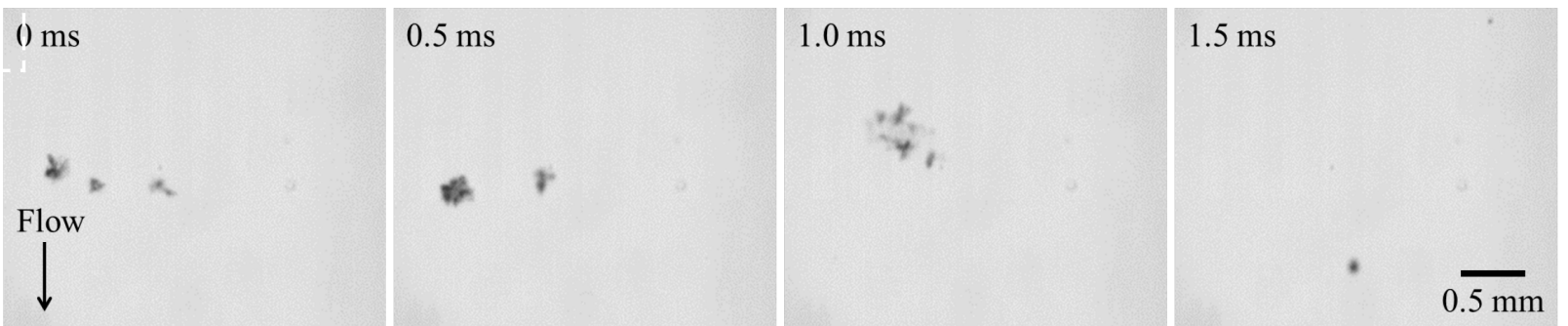

(b)
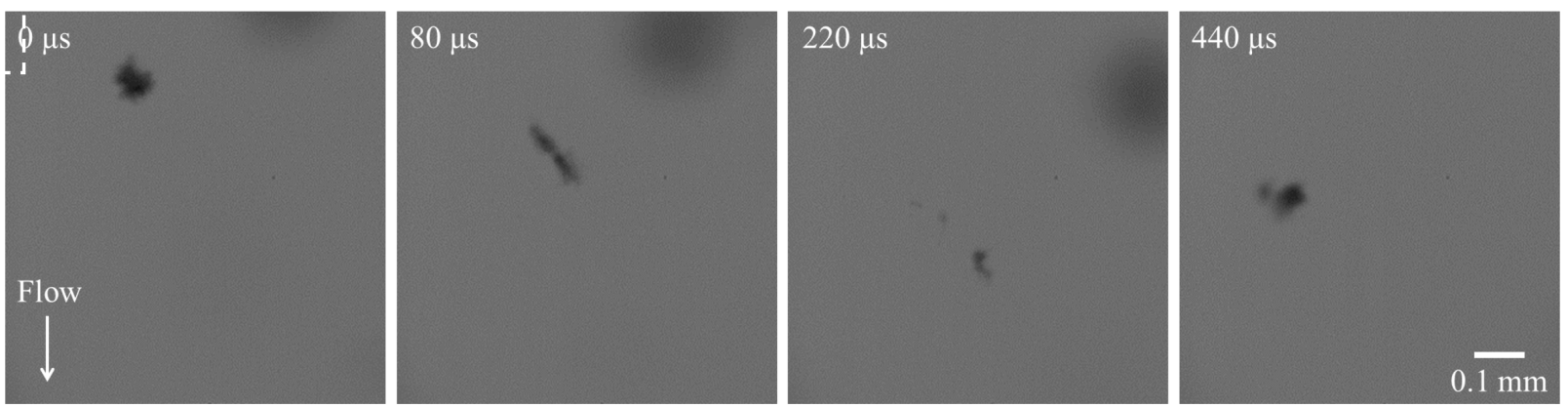

Figure 4: Sequential images of acoustic cavitation bubbles in the ultrasonic water flow through the square glass tube: (a) $0.43 \mathrm{MHz}$ ultrasound, (b) $0.95 \mathrm{MHz}$ ultrasound. 
(a)
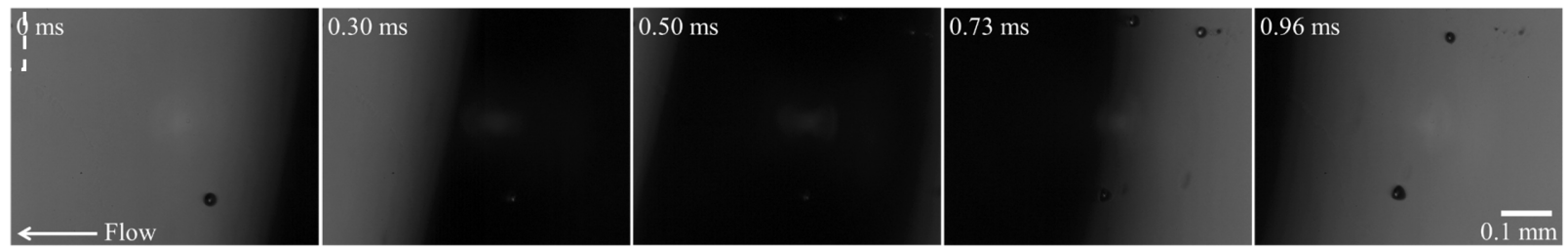

(b)

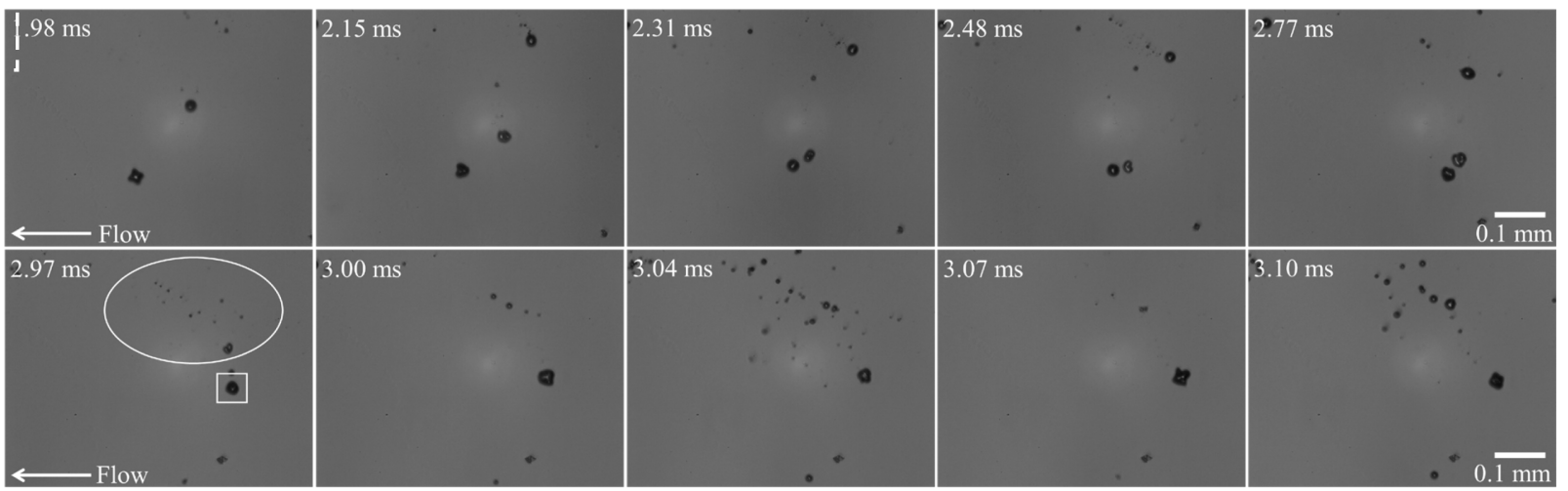

Figure 5: Sequential images of acoustic cavitation bubbles in the ultrasonic water film flow spreading over the glass surface for the case of the $0.43 \mathrm{MHz}$ ultrasound: (a) Evolution of the image darkness variation showing surface wave propagation at the interface between the liquid film and the ambient air, (b) Trapping, advection, and oscillation of cavitaiton bubbles after the surface wave passed by the observation spot. The radius of the larger bubble (white square) is about $15 \mu \mathrm{m}$ and that of the smaller bubbles (white circle) is about $3 \mu \mathrm{m}$.

(a)
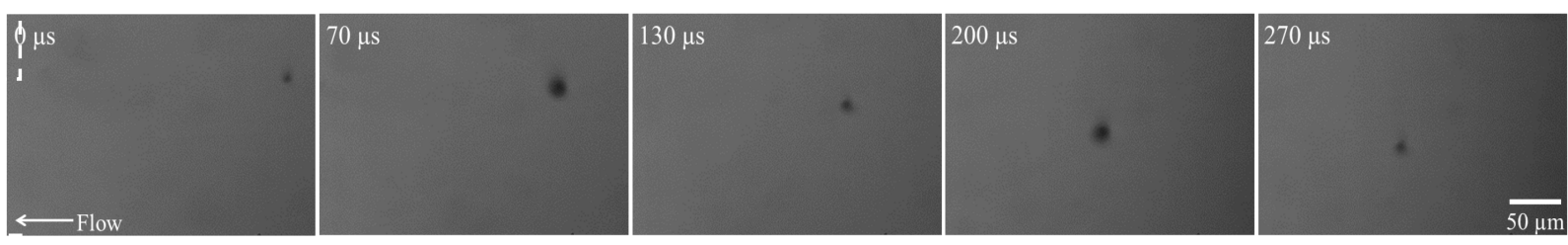

(b)
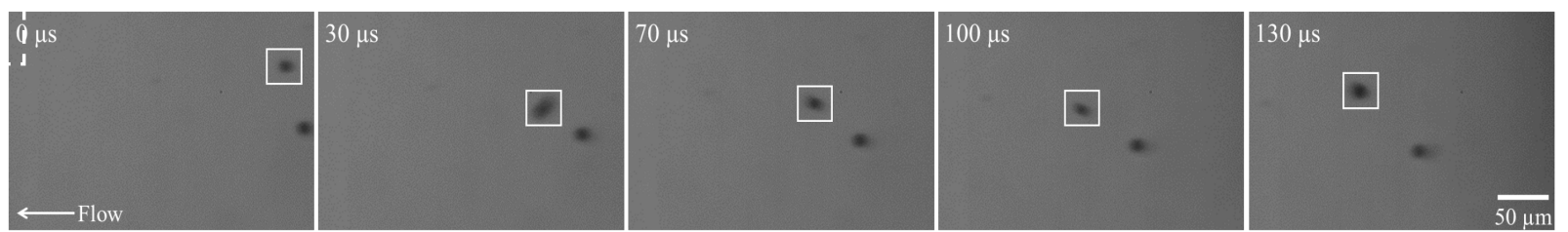

Figure 6: As in figure 5, but for the case of the $0.95 \mathrm{MHz}$ ultrasound. (a) An oscillating cavitation bubble whose instantaneous radius is between $3 \mu \mathrm{m}$ and $7 \mu \mathrm{m}$. (b) Advection of a cavitation bubble (white square) whose radius is about $5 \mu \mathrm{m}$.

\section{Summary}

In short, the schlieren and backlight visualization results suggest that the acoustic field and cavitation bubbles exist in the 0.43 and $0.95 \mathrm{MHz}$ ultrasonic water flow and the subsequent spreading film flow over the glass surface. That is, as in the case of conventional ultrasonic cleaning baths, cavitation bubbles will act as active cleaning agents in ultrasonic water flow cleaning.

\section{References}

[1] M. Hauptmann et al.: Microelectron. Eng. 87 (2010), p. 1512.

[2] W. Kim et al.: Appl. Phys. Lett. 94 (2009), p. 081908.

[3] T. Yamashita and K. Ando: Ultrason. Sonochem. 52 (2019), p. 268. 
[4] M. Minnaert: Philos. Mag. 16 (1933), p. 235.

[5] M. Devaud et. al.: Eur. J. Phys. 29 (2008), p. 1263.

[6] M. Ashokkumar et. al.: Ultrason. Sonochem. 14 (2007), p. 470.

[7] T. G. Leighton et. al.: Eur. J. Phys. 11 (1990), p. 47. 